\title{
Langmuir
}

This document is confidential and is proprietary to the American Chemical Society and its authors. Do not copy or disclose without written permission. If you have received this item in error, notify the sender and delete all copies.

\section{The headgroup (a)symmetry strongly determines the aggregation behavior of single-chain phenylene-modified bolalipids and their miscibility with classical phospholipids}

\begin{tabular}{|r|l|}
\hline Journal: & Langmuir \\
\hline Manuscript ID: & la-2014-01160s.R2 \\
\hline Manuscript Type: & Article \\
\hline Date Submitted by the Author: & n/a \\
\hline Complete List of Authors: & $\begin{array}{l}\text { Drescher, Simon; Martin-Luther-Universitaet Halle-Wittenberg, Institute of } \\
\text { Pharmacy } \\
\text { Lechner, Bob-Dan; Martin-Luther-Universitaet Halle-Wittenberg, Institute } \\
\text { of Chemistry } \\
\text { Garamus, Vasil; Helmholtz Zentrum Geesthacht (HZG), } \\
\text { Almasy, Laszlo; Wigner Research Centre, } \\
\text { Meister, Annette; Martin-Luther-Universitaet Halle-Wittenberg, Center for } \\
\text { structure and dynamics of proteins (MZP) } \\
\text { Blume, Alfred; Martin-Luther-Universitaet Halle-Wittenberg, Institute of } \\
\text { Chemistry }\end{array}$ \\
\hline
\end{tabular}

\section{SCHOLARONE ${ }^{\text {m }}$ \\ Manuscripts}




\section{The headgroup (a)symmetry strongly determines the}

\section{aggregation behavior of single-chain phenylene-modified}

\section{bolalipids and their miscibility with classical phospholipids}

Simon Drescher, ${ }^{* \dagger}$ Bob-Dan Lechner, ${ }^{\ddagger}$ Vasil M. Garamus, ${ }^{\text {,I }}$ László Almásy, ${ }^{\$}$ Annette Meister, ${ }^{\#}$ Alfred Blume $e^{\ddagger}$

$\dagger$ Institute of Pharmacy, Martin-Luther-Universitaet (MLU) Halle-Wittenberg, WolfgangLangenbeck-Str. 4, 06120 Halle (Saale), Germany; ${ }^{\ddagger}$ Institute of Chemistry, MLU HalleWittenberg, von-Danckelmann-Platz 4, 06120 Halle (Saale), Germany; ${ }^{\mathbb{I}}$ Helmholtz-Zentrum Geesthacht (HZG), Max-Planck-Str. 1, 21502 Geesthacht, Germany; ${ }^{\$}$ Wigner Research Centre for Physics, Budapest 1121 Konkoly Thege út 29, Hungary; ${ }^{\#}$ Center for structure and dynamics of proteins (MZP), MLU Halle-Wittenberg, Biocenter, Weinbergweg 22, 06120 Halle (Saale), Germany.

\section{KEYWORDS}

Bolalipids, Phospholipids, Aggregation Behavior, Self-Assembly, Mixing Behavior, Nanofibers, Micelles 


\begin{abstract}
In the present work, we describe the synthesis of two single-chain phenylene-modified bolalipids, namely PC-C17pPhC17-PC and PC-C17pPhC17-OH, with either symmetrical (phosphocholine) or asymmetrical (phosphocholine and hydroxyl) headgroups using a Sonogashira cross coupling reaction as key-step. The temperature-dependent aggregation behavior of both bolalipids in aqueous suspension was studied using transmission electron microscopy (TEM), differential scanning calorimetry (DSC), Fourier-transform infrared (FTIR) spectroscopy, small angle neutron scattering (SANS), and X-ray scattering. We show that different headgroup symmetries lead to a change in the aggregation behavior: Whereas $\mathrm{PC}-\mathrm{C} 17 \mathrm{pPhC} 17-\mathrm{PC}$ forms nanofibers with a diameter of $5.7 \mathrm{~nm}$ that transform into small ellipsoidal micelles at $23{ }^{\circ} \mathrm{C}$, the PC-C17pPhC17-OH self-assembles into lamellae with bolalipid molecules in an antiparallel orientation up to high temperatures. Furthermore, the mixing behavior of both bolalipids with bilayer forming phospholipids (DPPC and DSPC) was studied by means of DSC and TEM. The aim was to stabilize bilayer membranes formed of phospholipids in order to improve these mixed lipid vesicles for drug delivery purposes. We show that the symmetrical $\mathrm{PC}-\mathrm{C} 17 \mathrm{pPhC} 17-\mathrm{PC}$ is miscible with DPPC and DSPC, however, closed lipid vesicles are not observed and elongated micelles and bilayer fragments are found instead. In contrast, the asymmetrical PC-C17pPhC17$\mathrm{OH}$ shows no miscibility with phospholipids at all.
\end{abstract}

\title{
INTRODUCTION
}

Amphiphilic molecules with two hydrophilic headgroups attached to both ends of a long hydrocarbon spacer, consisting either of a single alkyl chain or two chains connected via a glycerol moiety, are called bolalipids. ${ }^{1}$ Bolalipids can be found in the membrane lipids of certain species 
of archaebacteria where they are responsible for the outstanding stability of those membranes against harsh external conditions, e.g., very high temperature or low $\mathrm{pH}$ values. ${ }^{2-4}$ Chemically pure representatives of naturally occurring symmetrical double-chain bolalipids and their artificial analogues ${ }^{5-9}$ mostly form lamellar, sheet-like and vesicular aggregates in aqueous suspension. On the other hand, symmetrical single-chain bolalipids composed of two large phosphocholine (PC) headgroups attached to one alkyl chain, e.g. PC-C32-PC, self-assemble in water into well-defined nanofibers, leading to an efficient gelation of the solvent. ${ }^{10}$ This selfassembly process is exclusively driven by hydrophobic interactions of the long alkyl chain, because the PC headgroups cannot form intermolecular hydrogen bonds. The nanofibers are composed of bolalipid molecules that are arranged side by side but twisted relative to each other due to the bulkiness of the PC headgroup in comparison to the small cross-sectional area of the alkyl chain. ${ }^{10}$ The twisting leads to a helical superstructure of the nanofiber, which was proved by high-resolution atomic force microscopy $(\mathrm{AFM})^{11}$ and coarse-grained off-lattice Monte Carlo simulations. ${ }^{12}$ Heating the hydrogel of a PC-C32-PC suspension above a distinct temperature leads to the reversible transformation of the nanofibers into small spherical micelles and the gel character is lost. ${ }^{10}$

By varying the chemical structure of these single-chain bolalipids the aggregation properties can be influenced and controlled in a desired way. To achieve this intention, two types of structural modifications are conceivable: (i) changes in the headgroup region and (ii) variations within the alkyl spacer chain. ${ }^{13}$ For instance, the shortening of the alkyl chain leads to a decrease of the fiber-micelle-transition and, hence, the gel-sol-conversion temperature. ${ }^{14}$ Moreover, since the self-assembly of PC-C32-PC in aqueous suspensions is exclusively driven by van-der-Waals interactions of the long alkyl chain, perturbations, such as the insertion of hetero atoms, ${ }^{15}$ 
acetylene groups and methyl branches ${ }^{13}$ within the spacer chain result in a pronounced destabilization of the fiber aggregates. On the other hand, modifications in the headgroup region can be obtained, e.g., by the reduction of the space requirement of the headgroup by a stepwise replacement of methyl moieties. This leads to a series of phosphodimethylethanolamines $\left(\mathrm{Me}_{2} \mathrm{PE}-\mathrm{Cn}-\mathrm{Me}_{2} \mathrm{PE}\right)$, which self-assemble into nanofibers ${ }^{16}$ or square lamellae, ${ }^{17}$ and the phosphomonomethylethanolamine MePE-C32-MePE that forms monolayer-like structures. ${ }^{18}$

A further way of modifications that induces changes in aggregation properties is the introduction of headgroup asymmetry, i.e., the coupling of two headgroups of different size to the same alkyl chain. This asymmetry leads to two different orientations of the bolalipids in lamellar structures, either parallel or antiparallel. A parallel orientation of the bolalipids provokes the formation of rods, nanotubes ${ }^{19-21}$ or nanotapes ${ }^{22}$ with, e.g., a different curvature of the inner and outer surface of the nanotube. On the other hand, an antiparallel (interdigitated) orientation of the bolalipid results in the formation of monolayer-like aggregates. ${ }^{20,23}$ Recently, we investigated the lyotropic behavior of DMAPPC-C32-OH, ${ }^{24}$ an asymmetrical bolalipid bearing a large 2-(dimethylaminopropyl)-PC (DMAPPC) headgroup and a small hydroxy moiety attached to a C32alkyl chain. This bolalipid self-assembles into short elongated micelles or lamellar structures, depending on the protonation state.

Another promising property of bolalipids is that they can be inserted in a stretched conformation into phospholipid bilayer membranes, leading to a stabilization of these phospholipid vesicles. This stabilizing approach was adopted from naturally occurring archaebacterial membrane lipids ${ }^{4,25,26}$ and its applicability to drug delivery systems for pharmaceutical purposes was confirmed for a variety of natural and artificial bolalipids. ${ }^{27-34}$

In our study we combine both, alkyl chain modifications and the introduction of headgroup 
asymmetry. At first, we synthesized two novel single-chain bolalipids, namely PC-C17pPhC17PC and PC-C17pPhC17-OH. Both bolalipids are composed of a central para-substituted phenyl ring within the alkyl chain and either two PCs headgroups (symmetrical) or a PC and hydroxy (asymmetrical) headgroup (see Figure 1).

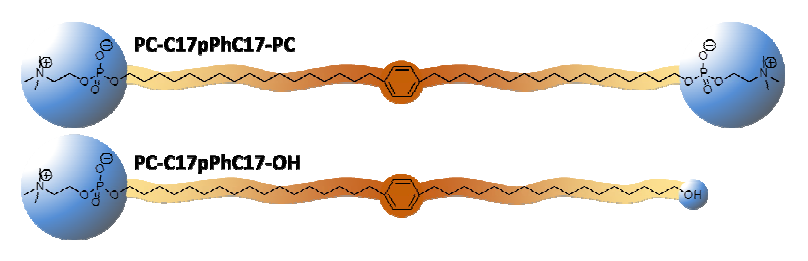

Figure 1. Chemical structure and schematic representation of single-chain phenylene-modified bolalipids $\mathrm{PC}-\mathrm{C} 17 \mathrm{pPhC} 17-\mathrm{PC}$ and $\mathrm{PC}-\mathrm{C} 17 \mathrm{pPhC} 17-\mathrm{OH}$ investigated in this work.

Secondly, the temperature-dependent aggregation behavior in aqueous suspension of the symmetrical bolalipid is compared to the self-assembly properties of its asymmetrical counterpart using differential scanning calorimetry (DSC), Fourier-transform infrared (FTIR) spectroscopy, transmission electron microscopy (TEM), small angle neutron scattering (SANS), and X-ray scattering. Thirdly, we investigated the miscibility of the novel bolalipids with bilayer forming 1,2-dipalmitoyl-sn-glycero-3-phosphocholine (DPPC) and 1,2-distearyl-sn-glycero-3phosphocholine (DSPC) by means of DSC and TEM. We will show that both, the alkyl chain modification and the headgroup asymmetry of the bolalipids, have a great impact on the aggregation behavior and also the miscibility with classical phospholipids.

\section{EXPERIMENTAL SECTION}

\section{Chemicals.}

1,2-Dipalmitoyl-sn-glycero-3-phosphocholine (DPPC) and 1,2-distearyl-sn-glycero-3-phosphocholine (DSPC) were purchased from Lipoid KG (Ludwigshafen, Germany).

\section{Syntheses.}


The synthetic procedures and the analytical data of the substances are described in detail in the Supporting Information (SI).

Methods.

Sample preparation. The appropriate amount of the bolalipid was suspended in $\mathrm{H}_{2} \mathrm{O}$ (MilliQ) and $\mathrm{D}_{2} \mathrm{O}$ (Sigma Aldrich), respectively. Homogeneous suspensions were obtained by heating to $90{ }^{\circ} \mathrm{C}$ and vortexing. Binary lipid mixtures were prepared from lipid stock solutions in $\mathrm{CHCl}_{3} / \mathrm{MeOH}(2 / 1, \mathrm{v} / \mathrm{v})$ as solvent by mixing appropriate volumes of the stock solutions. Afterwards, the organic solvent was removed in a stream of $\mathrm{N}_{2}$. The resulting lipid films were kept in an evacuated flask for $24 \mathrm{~h}$ to remove residual traces of solvent. The suspensions were then prepared by adding a certain volume of aqueous phosphate buffer $(10 \mathrm{mM}, \mathrm{pH}=7.4)$ to obtain a total lipid concentration of $3 \mathrm{mM}$. The samples were vigorously vortexed for $30 \mathrm{~min}$ at $60{ }^{\circ} \mathrm{C}$ to obtain a homogeneous suspension.

Differential Scanning Calorimetry (DSC). DSC measurements were performed using a MicroCal VP-DSC differential scanning calorimeter (MicroCal Inc. Northampton, MA, USA). Before the measurements, the sample suspension and the water (or phosphate buffer) reference were degassed under vacuum while stirring. A heating rate of $20 \mathrm{~K} \mathrm{~h}^{-1}$ was used, and the measurements were performed in the temperature interval from $2{ }^{\circ} \mathrm{C}$ to $95{ }^{\circ} \mathrm{C}$. To check the reproducibility, three consecutive scans were recorded for the sample. The water-water (bufferbuffer) baseline was subtracted from the thermogram of the sample, and the DSC scans were evaluated using MicroCal Origin 8.0 software.

Transmission Electron Microscopy (TEM). The negative stained samples were prepared by spreading $5 \mu \mathrm{L}$ of the bolalipid suspension $\left(c=0.05 \mathrm{mg} \mathrm{mL}^{-1}\right)$ onto a copper grid coated with a Formvar film. After $1 \mathrm{~min}$, excess liquid was blotted off with filter paper and $5 \mu \mathrm{L}$ of $1 \%$ 
aqueous uranyl acetate solution were placed onto the grid and drained off after $1 \mathrm{~min}$. Specimens prepared below ambient temperature $\left(T=5^{\circ} \mathrm{C}\right)$ were dried for 2 days at $5{ }^{\circ} \mathrm{C}$ and kept in an exsiccator at ambient temperature. Specimens prepared in a modified drying oven above ambient temperature were dried for $1 \mathrm{~h}$ at the appropriate temperature and kept in an exsiccator at ambient temperature. All specimens were examined with a Zeiss EM 900 transmission electron microscope (Carl Zeiss Microscopy GmbH, Oberkochen, Germany).

Fourier-transform Infrared Spectroscopy (FTIR). Infrared spectra were collected on a Bruker Vector 22 Fourier transform spectrometer with DTGS detector operating at $2 \mathrm{~cm}^{-1}$ resolution. Sample $\left(c=50 \mathrm{mg} \mathrm{mL}^{-1}\right.$ in $\left.\mathrm{D}_{2} \mathrm{O}\right)$ were placed between two $\mathrm{CaF}_{2}$ windows, separated by a $56 \mu \mathrm{m}$ spacer. IR spectra were recorded in steps of $2 \mathrm{~K}$ in the temperature range from $9{ }^{\circ} \mathrm{C}$ to $75^{\circ} \mathrm{C}$. After an equilibration time of $8 \mathrm{~min}, 64$ scans were recorded and accumulated. The corresponding spectra of the solvent $\left(\mathrm{D}_{2} \mathrm{O}\right)$ were subtracted from the sample spectra using the OPUS software supplied by Bruker.

Small angle neutron scattering (SANS). SANS measurements were performed on the Yellow Submarine instrument at the BNC in Budapest (Hungary). ${ }^{35}$ The overall $q$-range was from $0.03 \mathrm{~nm}^{-1}$ to $1.0 \mathrm{~nm}^{-1}$. The samples were filled in Hellma quartz cells of $2 \mathrm{~mm}$ path length and placed in a thermostated holder kept with accuracy $\pm 0.5^{\circ} \mathrm{C}$. The raw scattering patterns were corrected for sample transmission, room background, and sample cell scattering. The twodimensional scattering patterns were azimuthally averaged, converted to an absolute scale and corrected for detector efficiency dividing by the incoherent scattering spectra of $1 \mathrm{~mm}$ thick pure water. The scattering from $\mathrm{D}_{2} \mathrm{O}$ was subtracted as the background.

$X$-ray scattering. Powder patterns were measured in transmission with a stationary linear position sensitive detector $\left(2 \Theta=0-44^{\circ}\right)$ on a stage including primary $\operatorname{Ge}(111)$ monochromator 
and high temperature attachment (STOE \& CIE, Darmstadt, Germany). The bolalipid samples (50 wt.\% $\left.\mathrm{H}_{2} \mathrm{O}\right)$ were sealed in glass capillaries (diameter $\left.1.5 \mathrm{~mm}\right) . \mathrm{CuK}_{\alpha 1}(\lambda=0.154051 \mathrm{~nm})$ radiation was used, and the scattering was corrected with respect to a capillary filled with $\mathrm{H}_{2} \mathrm{O}$ $\left(I_{\text {norm }}=I_{\text {sample }} / I_{\text {cap }}\right)$. The X-ray patterns were combined in a single contour diagram to continuously present the scattered intensity from the SAXS to the WAXS region $\left(2 \Theta=0-44^{\circ}\right.$, $\mathrm{s}=0-4.7 \mathrm{~nm}^{-1}$ ) between $-30{ }^{\circ} \mathrm{C}$ and $135^{\circ} \mathrm{C}$ in steps of $2 \mathrm{~K}$. The heating rate was $1 / 15 \mathrm{~K} \mathrm{~min}^{-1}$ ( 5 min equilibration, 10 min exposition for each pattern) for the applied temperature protocol.

\section{RESULTS AND DISCUSSION}

\section{Syntheses of bolalipids}

For the synthesis of the symmetrical bolalipid we converted the phenylene-modified 1, $\omega$-diol, which was synthesized using the bis-Sonogashira cross-coupling reaction described previously, ${ }^{36}$ into the PC-C17pPhC17-PC by established phosphorylation and quarternisation reactions (see SI, Scheme S1, left panel). For the synthesis of the asymmetrical PC-C17pPhC17-OH we used a similar Sonogashira cross-coupling reaction as key step and HO-C15pAcPh-Br and Ac-C15-OBn as starting materials. The Ac-C15-OBn can be prepared in three steps from THPO-C15-Br ${ }^{14}$ as detailed in the SI. The Sonogashira cross-coupling of HO-C15pAcPh-Br with Ac-C15-OBn was carried out under comparable conditions as described for the bis-coupling reaction, ${ }^{36}$ and the HO-C15pAcPhAcC15-OBn was obtained in $61 \%$ yield after chromatographic purification. This mono-protected diol was then transformed into the bolalipid PC-C15pAcPhAcC15-OBn by phosphorylation and quarternisation reaction. ${ }^{14}$ The final step combined the cleavage of the Bn protecting group and the hydrogenation of the triple bonds, resulting in the formation of the asymmetrical bolalipid PC-C17pPhC17-OH (Scheme S1, right panel). 
The stepwise performed Sonogashira cross-coupling reaction of dibromo benzene derivatives with various, orthogonally protected alkynols is a promising method for the preparation of phenylene-modified 1, $\omega$-diols and, in continuation, asymmetrical bolaphospholipids. Hence, this synthetic pathway is a valuable amendment to the synthesis of phenylene-modified diols using the Grignard homo-coupling reactions published previously. ${ }^{37}$

\section{Temperature-dependent aggregation behavior of pure bolalipids in aqueous suspension}

Symmetrical single-chain bolalipids without chain modifications mostly aggregate in water into long, flexible nanofibers resulting in the formation of a transparent hydrogel. A temperature increase leads to the transformation of the fibers into small spherical micelles and the liquefaction of the gel. This transition is reversible and it can be monitored by DSC, TEM, and FTIR. $^{10}$ In the DSC thermogram of, e.g., PC-C32-PC two endothermic transitions $\left(T_{m}\right)$ were observed, the first one at $48.7^{\circ} \mathrm{C}$ that is attributed to the breakdown of the nanofibers and the second one at about $73{ }^{\circ} \mathrm{C}$ representing a transition between two different types of micelles. ${ }^{14}$

TEM:

When suspended in water, the bolalipid PC-C17pPhC17-PC $\left(c=1 \mathrm{mg} \mathrm{mL}^{-1}\right)$ forms a transparent hydrogel after storage in the refrigerator at $4{ }^{\circ} \mathrm{C}$ (see Figure S1). This behavior indicates a self-assembly of $\mathrm{PC}-\mathrm{C} 17 \mathrm{pPhC} 17-\mathrm{PC}$ into nanofibers as observed for similar phenylene-free bolalipids. However, the hydrogel of $\mathrm{PC}-\mathrm{C} 17 \mathrm{pPhC} 17-\mathrm{PC}$ is not as stable as hydrogels formed of, e.g., PC-C32-PC. In contrast, the asymmetrical bolalipid PC-C17pPhC17$\mathrm{OH}$ forms no hydrogel. Instead, the formation of an opalescent suspension after several heating and vortexing cycles was observed (see Figure S1). To visualize the structures of aggregates formed in the aqueous suspensions at $20^{\circ} \mathrm{C}$ TEM images were obtained from both negatively stained samples (Figure 2). 


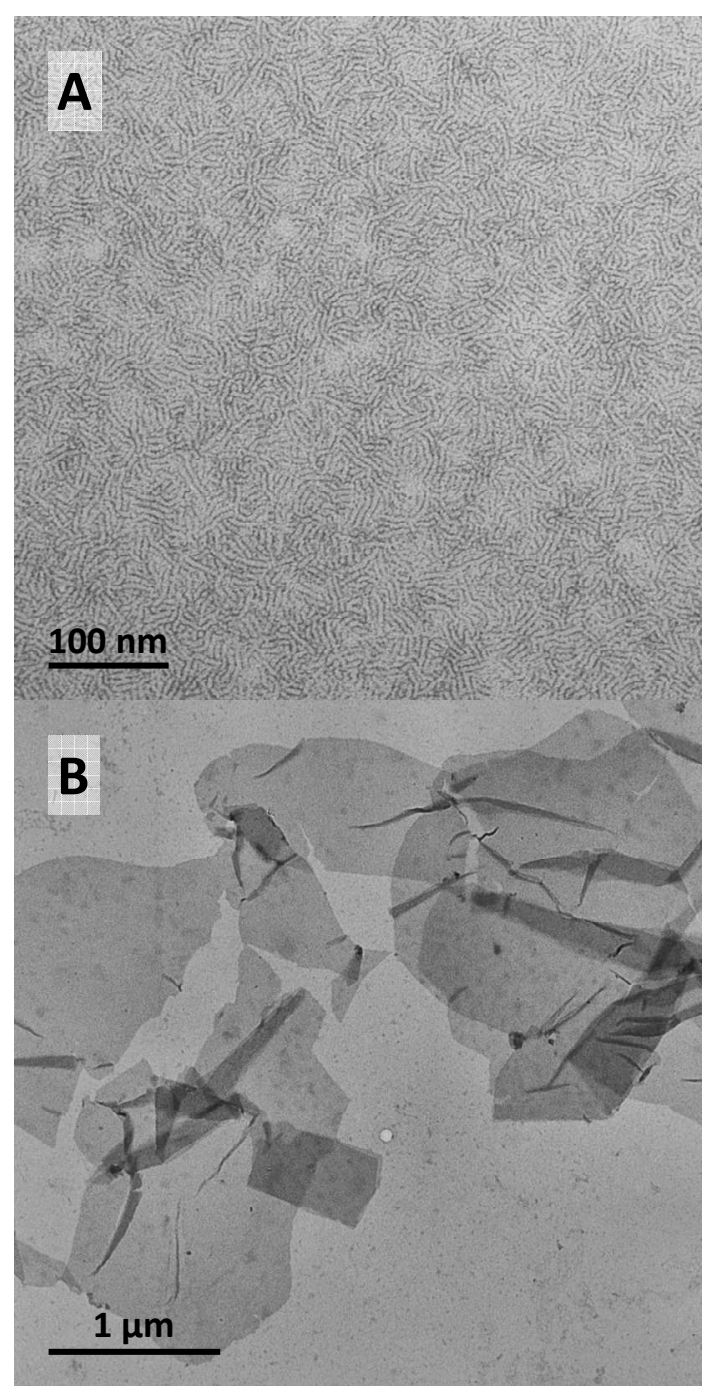

Figure 2. TEM images of aqueous suspension $\left(c=0.05 \mathrm{mg} \mathrm{mL}^{-1}\right)$ of (A) PC-C17pPhC17-PC and (B) $\mathrm{PC}-\mathrm{C} 17 \mathrm{pPhC} 17-\mathrm{OH}$. The samples were prepared at $20{ }^{\circ} \mathrm{C}$ and stained with uranyl acetate.

The TEM image of the symmetrical PC-C17pPC17-PC (Figure 2A) shows the presence of densely packed nanofibers. The thickness of the nanofibers is 5-6 nm, which roughly corresponds to the length of the bola molecule. A similar symmetrical bolalipid, namely PCC16pPhC16-PC ${ }^{37}$ with a slightly shorter alkyl chain (two methylene units) compared to the bolalipid described here, shows the formation of small elongated micelles in aqueous suspension at room temperature. These micelles have a size between $8 \mathrm{~nm}$ and $18 \mathrm{~nm}$ in length and $3-4 \mathrm{~nm}$ in width. However, the formation of nanofibers and, hence, a gelation of the solvent were not 
observed for the PC-C16pPhC16-PC sample. This indicates that the tendency to form fibrous aggregates depends critically on the over-all alkyl chain length of the phenylene-modified bolalipids: The shortening of the alkyl chain by two methylene units is sufficient to reduce the van-der-Waals contacts of neighboring alkyl chains in such a manner that the formation of long nanofibers is not possible.

In contrast to the symmetrical bolalipid, the TEM image of the asymmetrical counterpart PC$\mathrm{C} 17 \mathrm{pPhC17-OH}$ (Figure 2B) shows the presence of large lamellar structures. These sheet-like aggregates that in the TEM image appear folded and partly disrupted have a size of about several micrometers. The folding is due to the drying process during the EM sample preparation. In consequence, the aggregation behavior of phenylene-modified bolalipids can be tuned by simple changes in the headgroup asymmetry, i.e., the replacement of one PC headgroup of the bolalipid by a small hydroxy moiety. A comparable behavior was observed previously for phenylene-free bolalipids with a larger difference in the size of the two headgroups attached at one alkyl chain. ${ }^{24}$ DSC and FTIR:

To investigate the temperature-dependent aggregation behavior of both bolalipids in aqueous suspension, DSC and FTIR measurements were carried out (Figure 3).
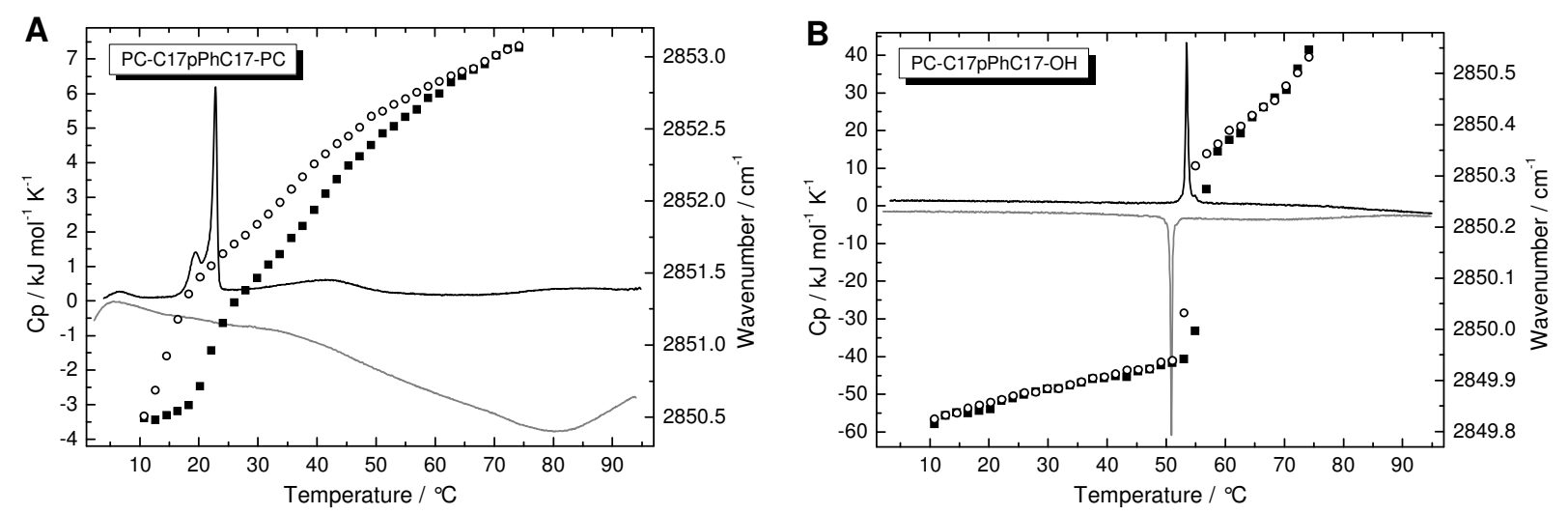

Figure 3. DSC data (solid lines, $c=1 \mathrm{mg} \mathrm{mL}^{-1}$ in $\mathrm{H}_{2} \mathrm{O}$, heating: black, cooling: grey) and FTIR spectroscopic data (symmetric methylene stretching vibration, scattered data, $c=50 \mathrm{mg} \mathrm{mL}^{-1}$ in 
$\mathrm{D}_{2} \mathrm{O}$, heating: filled squares, cooling: open circles) of (A) $\mathrm{PC}-\mathrm{C} 17 \mathrm{pPhC} 17-\mathrm{PC}$ and (B) PC$\mathrm{C} 17 \mathrm{pPhC} 17-\mathrm{OH}$.

The DSC heating curve of the PC-C17pPhC17-PC shows three endothermic peaks (Figure 3A): a very small peak at $6.6^{\circ} \mathrm{C}$, a second one at $22.8^{\circ} \mathrm{C}$ that probably corresponds to a fibermicelle-transition, and a third very broad peak between $40-42{ }^{\circ} \mathrm{C}$, which can be attributed to a transition between two different types of micelles. In contrast, the similar bolalipid PCC16pPhC16-PC showed only the formation of small micelles at room temperature and no transition was observed in the DSC in the temperature range between $2-95{ }^{\circ} \mathrm{C}$ (see Figure $\mathrm{S} 2) .{ }^{13,37}$ The corresponding cooling curve of the $\mathrm{PC}-\mathrm{C} 17 \mathrm{pPhC} 17-\mathrm{PC}$ suspension shows no transition peaks, which is an indication for a hindered reformation of the fibrous aggregates on the time scale of the cooling process. As mentioned above, for the formation of the nanofibers the sample has to be kept at $4{ }^{\circ} \mathrm{C}$ for prolonged periods of time. Since the DSC scans of suspensions of the unmodified PC-C32-PC ${ }^{14}$ and PC-C34-PC ${ }^{11}$ show a very cooperative fibermicelle-transition at $48.7^{\circ} \mathrm{C}$ and $56.5^{\circ} \mathrm{C}$, the decreased transition temperature for PC$\mathrm{C} 17 \mathrm{pPhC17-PC}$ reflects the strong influence of the phenyl ring in the middle part of the alkyl chain on the van-der-Waals interactions between neighboring alkyl chains and the perturbation of optimal chain packing necessary for the formation of stable nanofibers. This phenomenon was also described for other alkyl chain modifications, such as the insertion of sulfur atoms, ${ }^{15}$ acetylene groups, and methyl branches. ${ }^{13}$ The fact that the shorter chain analogue PC-C16pPhC16-PC forms only micelles reveals the existence of a critical length for the unmodified alkyl chain, below which a formation of nanofibers is not possible any more. To investigate the structure of aggregates formed in aqueous suspension below and above $T_{m}$ of PC-C17pPhC17-PC, samples for TEM were also prepared at $4{ }^{\circ} \mathrm{C}$ and $35^{\circ} \mathrm{C}$, respectively. The TEM images (see Figures S3, S4) show the presence of flexible nanofibers below $T_{m}$ and worm-like micelles above $T_{m}$. These 
micelles are comparable to the aggregates formed of $\mathrm{PC}-\mathrm{C} 16 \mathrm{pPhC} 16-\mathrm{PC}$ at room temperature. ${ }^{37}$

In contrast to the symmetrical bolalipid PC-C17pPhC17-PC, the DSC heating curve of the asymmetrical PC-C17pPhC17-OH shows only one endothermic and very cooperative transition at $53.4{ }^{\circ} \mathrm{C}$ in the temperature range up to $95^{\circ} \mathrm{C}$ (Figure 3B). The corresponding cooling curve reveals the same peak with a small hysteresis of $2.5 \mathrm{~K}$.

To obtain information on the chain conformation of the bolalipid molecules inside the formed aggregates, IR spectra of suspensions of both bolalipids $\left(c=50 \mathrm{mg} \mathrm{mL}^{-1}\right.$ in $\left.\mathrm{D}_{2} \mathrm{O}\right)$ were recorded in the temperature range between $9-75^{\circ} \mathrm{C}$. The wavenumbers of the symmetric $\left(v_{s} \mathrm{CH}_{2}\right)$ and antisymmetric $\left(v_{a s} \mathrm{CH}_{2}\right)$ methylene stretching vibrational bands provide information about the conformational order of the alkyl chain. ${ }^{38,39}$ The temperature dependency of the wavenumbers of both methylene stretching vibrational bands of $\mathrm{PC}-\mathrm{C} 17 \mathrm{pPhC} 17-\mathrm{PC}$ and $\mathrm{PC}-\mathrm{C} 17 \mathrm{pPhC} 17-\mathrm{OH}$ is depicted in Figure 3, and in the SI in Figures S5, S6.

For the symmetrical PC-C17pPhC17-PC, the frequency of the bands at low temperatures are at $2850.5 \mathrm{~cm}^{-1}$ and $2919.8 \mathrm{~cm}^{-1}$ for $v_{s} \mathrm{CH}_{2}$ (Figure 3A) and $v_{a s} \mathrm{CH}_{2}$ (Figure S5), respectively, and indicate ordered alkyl chains in all-trans conformation. The frequencies are slightly higher compared to the $\mathrm{CH}_{2}$-stretching frequencies of the unmodified PC-C32-PC $\left(2849.6 \mathrm{~cm}^{-1}\right.$ and $\left.2918.6 \mathrm{~cm}^{-1}\right)^{10}$ due to the reduced van-der-Waals contacts of the phenylene-modified alkyl chains and/or to the overall shorter alkyl chains of PC-C17pPhC17-PC compared to PC-C32-PC. The wavenumber of both bands increases to $2853.1 \mathrm{~cm}^{-1}\left(v_{s} \mathrm{CH}_{2}\right)$ and $2924.3 \mathrm{~cm}^{-1}\left(v_{a s} \mathrm{CH}_{2}\right)$ at $75^{\circ} \mathrm{C}$. This increase in wavenumber, which is most pronounced in the temperature range around $T_{m}$ (at $23{ }^{\circ} \mathrm{C}$ ), is attributed to an increased amount of gauche conformers and, hence, a more flexible alkyl chain. The cooling curve shows nearly the same pattern for both stretching vibrational bands except a hysteresis of about $5 \mathrm{~K}$ indicating a delayed reformation of the ordered fibrous 
structures. This hysteresis is not visible in the DSC cooling scan due to the higher bolalipid concentration in the DSC experiment compared to the samples used for FTIR, as the kinetics for reformation of the nanofibers from micelles is, of course, concentration dependent.

For the asymmetrical $\mathrm{PC}-\mathrm{C} 17 \mathrm{pPhC} 17-\mathrm{OH}$ the wavenumbers of both methylene stretching vibrational bands occur at $2849.8 \mathrm{~cm}^{-1}\left(v_{s} \mathrm{CH}_{2}\right.$, Figure $\left.3 \mathrm{~B}\right)$ and $2917.0 \mathrm{~cm}^{-1}\left(v_{a s} \mathrm{CH}_{2}\right.$, see Figure S6). These values are slightly lower compared to the $\nu \mathrm{CH}_{2}$-bands of the symmetrical counterpart indicating more highly ordered alkyl chains. During heating, the wavenumbers of both bands increase to $2850.4 \mathrm{~cm}^{-1}\left(v_{s} \mathrm{CH}_{2}\right)$ and $2919.0 \mathrm{~cm}^{-1}\left(v_{a s} \mathrm{CH}_{2}\right)$ above $T_{m}\left(53{ }^{\circ} \mathrm{C}\right)$. Both frequency values indicate that the alkyl chain is still in an all-trans conformation, i.e., no "melting" of the alkyl chain occurs at this temperature.

To investigate the nature of this transition, the methylene scissoring vibrational band $\left(\delta \mathrm{CH}_{2}\right)$ was analyzed. The temperature dependent spectra for both bolalipids are shown in the SI (see Figures S7, S8): At low temperatures one observes a band at ca. $1471.5 \mathrm{~cm}^{-1}$ for the symmetrical and $1472.1 \mathrm{~cm}^{-1}$ for the asymmetrical bolalipid. For both bolalipids the bands decrease in intensity and shift to lower wavenumbers with increasing temperature. At the main transition temperature, for $\mathrm{PC}-\mathrm{C} 17 \mathrm{pPhC} 17-\mathrm{PC}$ a shift to $1468.0 \mathrm{~cm}^{-1}$ is observed when the nanofibers convert into micelles. For $\mathrm{PC}-\mathrm{C} 17 \mathrm{pPhC} 17-\mathrm{OH}$ the shift occurs to $1467.5 \mathrm{~cm}^{-1}$. For the asymmetrical bolalipid $\mathrm{PC}-\mathrm{C} 17 \mathrm{pPhC} 17-\mathrm{OH}$ this change in frequency is conceivably related to different chain packing modes in the ordered lamellar phases. However, in contrast to previous results found for the lamellar phase of an asymmetric phenylene-free bolalipid, ${ }^{24}$ a splitting of the methylene deformational band was not observed at low temperature indicating an orthorhombic perpendicular packing of the alkyl chains. ${ }^{40-43}$ Obviously, the additional phenyl ring in the middle part of the alkyl chain leads to some distortion and the alkyl chains cannot come in a 
very close and highly ordered contact required for the appearance of this splitting. Nevertheless, the change in frequency of the $\mathrm{CH}_{2}$-stretching bands and the $\mathrm{CH}_{2}$-deformation band indicates a very cooperative change in chain packing at this temperature. It is conceivable that the chains gain rotational freedom but are still in an all-trans conformation.

SANS and X-ray:

To get further insight into the structure of aggregates formed of both bolalipids, SANS and Xray scattering measurements were performed. At first, a suspension of the symmetrical PCC17pPhC17-PC ( $c=2 \mathrm{mg} \mathrm{mL}^{-1}$ in $\left.\mathrm{D}_{2} \mathrm{O}\right)$ was investigated by SANS at different temperatures. The scattering data and their fits are shown in Figure 4. The fits were performed using the IFT method described before ${ }^{16,18,44,45}$ and they are in quite good agreement with the experimental data. For fitting the scattering data obtained at $12{ }^{\circ} \mathrm{C}$, the model of infinitely long cylinders was used and for the fits of the scattering curves obtained at $28{ }^{\circ} \mathrm{C}$ and $55^{\circ} \mathrm{C}$ spherical aggregates were assumed. The results of the fits are provided in Table 1.

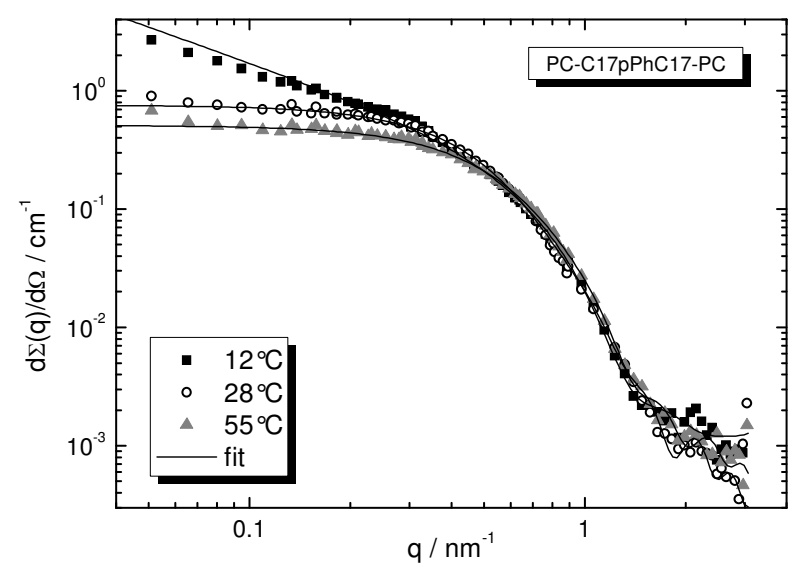

Figure 4. SANS data (symbols) and IFT fits (solid lines) of an aqueous suspension of PC$\mathrm{C} 17 \mathrm{pPhC17}-\mathrm{PC}\left(\mathrm{c}=2 \mathrm{mg} \mathrm{mL}^{-1}\right.$ in $\left.\mathrm{D}_{2} \mathrm{O}\right)$ measured at different temperatures. 
Table 1. SANS data obtained from IFT fits for aqueous suspensions of $\mathrm{PC}-\mathrm{C} 17 \mathrm{pPhC} 17-\mathrm{PC}$ in $\mathrm{D}_{2} \mathrm{O}$ at different temperatures. ${ }^{a}$ Data for PC-C32-PC and PC-C36-PC are shown for comparison.

\begin{tabular}{|c|c|c|c|c|c|c|c|}
\hline $\begin{array}{c}\text { Bolalipid } \\
\text { (concentration) }\end{array}$ & $T\left[{ }^{\circ} \mathrm{C}\right]$ & $\begin{array}{l}\text { Aggregate } \\
\text { shape }\end{array}$ & $\begin{array}{l}D_{\max } \\
{[\mathrm{nm}]}\end{array}$ & $\begin{array}{c}M[\mathrm{~g}] \text { or } \\
M_{L}\left[\mathrm{~g} \mathrm{~cm}^{-1}\right]\end{array}$ & $\begin{array}{l}N_{a g g}\left[\mathrm{~nm}^{-1}\right] \\
\text { or } N_{\text {agg }} \text { per } \\
\text { micelle }\end{array}$ & $\begin{array}{c}R_{g} \text { or } \\
R_{S C, g}[n m]\end{array}$ & $R[\mathrm{~nm}]$ \\
\hline \multirow{3}{*}{$\begin{array}{c}P C-C 17 p P h C 17-P C \\
\left(c=2 m g ~ m L^{-1}\right)\end{array}$} & 12 & fibers & 6.5 & $9.35 \cdot 10^{-14}$ & $6 \pm 1$ & $2.01 \pm 0.02$ & $2.84 \pm 0.02$ \\
\hline & 28 & micelles & 11.0 & $1.21 \cdot 10^{-19}$ & $79 \pm 4$ & $3.77 \pm 0.05$ & $4.87 \pm 0.05$ \\
\hline & 55 & micelles & 10.0 & $8.57 \cdot 10^{-20}$ & $56 \pm 3$ & $3.30 \pm 0.02$ & $4.26 \pm 0.02$ \\
\hline \multirow{2}{*}{$\begin{array}{c}P C-C 32-P C^{16} \\
\left(c=1 m g m L^{-1}\right)\end{array}$} & 25 & fibers & 4.5 & $1.47 \cdot 10^{-13}$ & $10 \pm 1$ & $1.51 \pm 0.02$ & $2.14 \pm 0.02$ \\
\hline & 60 & micelles & 7.5 & $1.05 \cdot 10^{-19}$ & $77 \pm 3$ & $2.46 \pm 0.02$ & $3.18 \pm 0.02$ \\
\hline \multirow{2}{*}{$\begin{array}{c}P C-C 36-P C^{17} \\
\left(c=1 m g m L^{-1}\right)\end{array}$} & 25 & fibers & 5.5 & $1.46 \cdot 10^{-13}$ & $10 \pm 1$ & $1.86 \pm 0.02$ & $2.63 \pm 0.01$ \\
\hline & 70 & micelles & 7.5 & $9.87 \cdot 10^{-20}$ & $77 \pm 4$ & $2.71 \pm 0.02$ & $3.50 \pm 0.02$ \\
\hline
\end{tabular}

${ }^{a} \mathrm{D}_{\text {max }}$ : maximal size or cross-section of aggregate , M: mass, $\mathrm{M}_{\mathrm{L}}$ : mass per unit length, $\mathrm{N}_{\mathrm{agg}}$ : aggregation number, $\mathrm{R}_{\mathrm{g}}$ : radius of gyration, $\mathrm{R}_{\mathrm{SC}, \mathrm{g}}$ : radius of gyration of cross-section, $\mathrm{R}$ and $\mathrm{RSC}$ are effective radius of aggregate or radius of cylindrical cross section in homogeneous approximation.

The results of the fits support the interpretation of the aggregate structure of PC-C17pPhC17PC as fibers below and micelles above the main DSC transition peak. The mean diameter $(d)$ of the bola fibers $(d=5.7 \mathrm{~nm})$ is in accordance with the one determined from TEM and corresponds roughly to the length of the bola molecule. If the additional phenyl ring in the middle part of the alkyl chain is set to a length of roughly $4 \mathrm{C}-\mathrm{C}$ bonds, PC-C17pPhC17-PC has an alkyl chain length equal to a $\mathrm{C} 38$-chain. Hence, the diameter of the PC-C17pPhC17-PC nanofiber fits well into the series of bola fibers composed of unmodified C32- $(\mathrm{PC}-\mathrm{C} 32-\mathrm{PC}, d=4.3 \mathrm{~nm} \text {; Table } 1)^{16}$ and C36-bolalipid molecules (PC-C36-PC, $d=5.3 \mathrm{~nm}) .{ }^{17}$ Modelling the scattering curves assuming stiff cylinders with an elliptical cross-section leads to values for the semi axes of $2.1 \pm$ $0.1 \mathrm{~nm}$ and $3.4 \pm 0.1 \mathrm{~nm}$ (axial ratio about 1.6, see Table S2). The PC-C17pPhC17-PC nanofibers have a lower aggregation number $N_{a g g} \mathrm{~nm}^{-1}$ compared to the unmodified bolalipids ( 6 vs. 10; see Table 1). It is obvious that the insertion of a phenyl ring in the middle part of the alkyl chain increases the space requirement (volume) of the alkyl chain as well as the whole bola molecule and, hence, decreases $N_{\text {agg }} \mathrm{nm}^{-1}$. 
As mentioned before, small micellar aggregates are observed for the aqueous PC$\mathrm{C} 17 \mathrm{pPhC} 17-\mathrm{PC}$ suspension at temperatures above $T_{m}$. At $28^{\circ} \mathrm{C}$ we obtain a mean diameter of the micelles of $9.7 \mathrm{~nm}$ and a $N_{\text {agg }}$ (number of molecules per micelle) of 79 . Modelling of the scattering curves by ellipsoids of revolution yields values for the semi axes of $a=b=3.2 \mathrm{~nm}$ and $c=8.2 \mathrm{~nm}$ (see Table S2). Compared to the unmodified bolalipids the mean diameter is slightly larger, which is due to the additional phenyl ring within the alkyl chain and an over-all longer alkyl chain, but the aggregation numbers are similar. Above the very broad DSC transition between $40-42{ }^{\circ} \mathrm{C}, d$ and $N_{\text {agg }}$ decrease to $8.5 \mathrm{~nm}$ and 56 due to a higher amount of gauche conformers within the alkyl chain. This change in the aggregate size of two different types of micelles was also found for aggregates formed of PC-C26-PC with an unmodified alkyl chain of 26 methylene units. ${ }^{18}$ However, the arrangement of the bolalipid molecules within these different types of micellar aggregates remains still unclear at this time.

The suspension of the asymmetrical PC-C17pPhC17-OH was not investigated by SANS as large lamellar aggregates are formed with highly ordered chains. X-ray diffraction measurements at different temperatures were performed instead, to gain information about the molecular packing and structural organization of the bolalipid molecules within the lamellar aggregates. The results are shown in Figure 5 as a contour plot. 


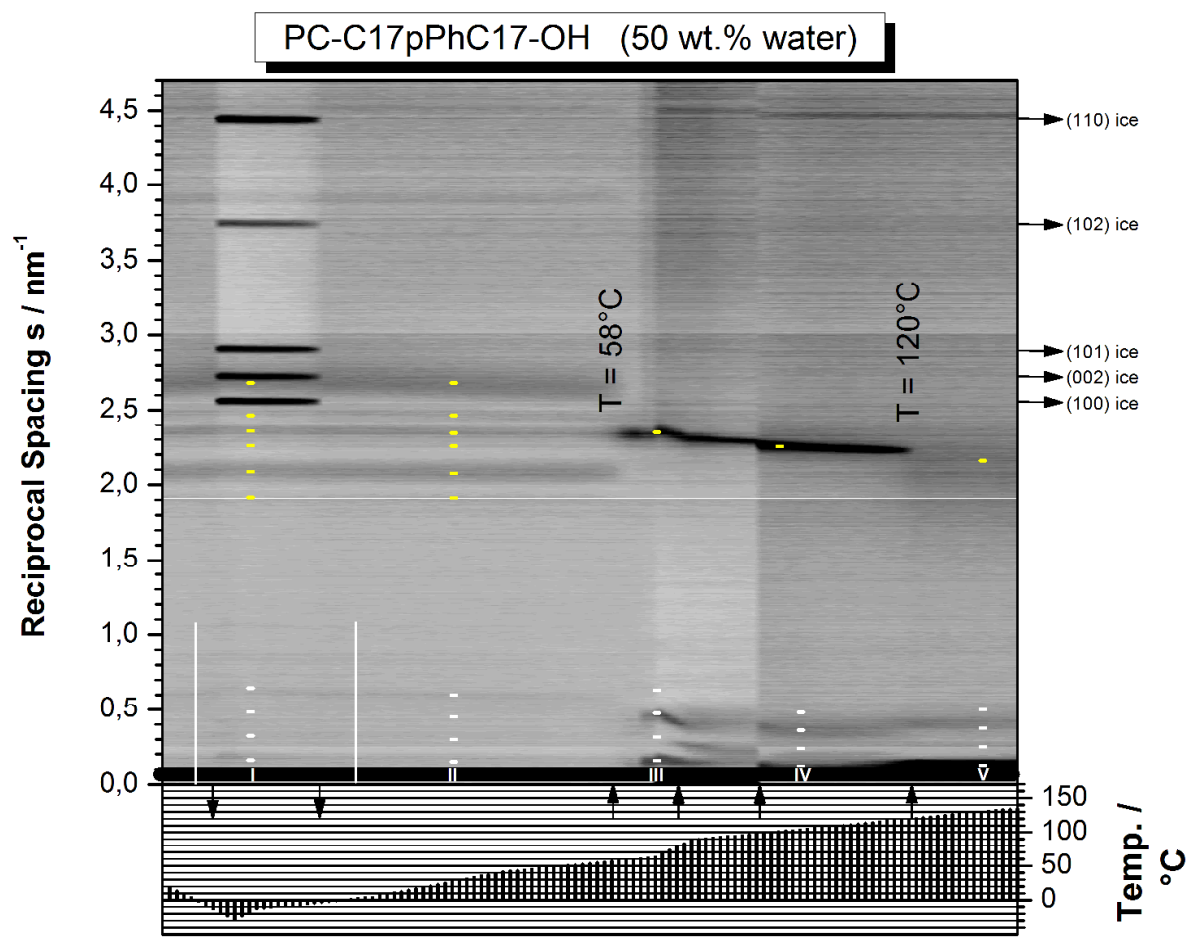

Relative Time / a. u.

Figure 5. X-ray contour diagram of a $50 \mathrm{wt} . \% \mathrm{PC}-\mathrm{C} 17 \mathrm{pPhC} 17-\mathrm{OH}$ sample in water. The scattering intensities are shown in the upper part as a function of reciprocal lattice spacing (ordinate) and temperature (abscissa). In the lower part the temperature course during the experiment is shown as a ramp. The two arrows pointing down indicate the onset of freezing and melting of water. In the temperature range between these arrows additional intense ice reflections are seen. The arrows pointing upwards indicate the transition temperatures of the bolalipid in the up and down scans. The intensities in the diagram have been scaled differently in the different temperature ranges, visible by the changes in gray scaling of the background intensity. Long spacings (different orders of the repeat distance, white dashes) and respective short spacings (fingerprint scattering due to the aliphatic chain packing, yellow dashes) belong together at the selected temperatures I-V: (I) $d=6.21 \mathrm{~nm}\left(s=0.161 \mathrm{~nm}^{-1},-18{ }^{\circ} \mathrm{C}\right)$, (II) $d=6.66 \mathrm{~nm}(s=$ $\left.0.150 \mathrm{~nm}^{-1}, 20^{\circ} \mathrm{C}\right)$, (III) $d=6.32 \mathrm{~nm}\left(s=0.158 \mathrm{~nm}^{-1}, 70^{\circ} \mathrm{C}\right)$, (IV) $d=8.33 \mathrm{~nm}\left(s=0.120 \mathrm{~nm}^{-1}\right.$, $\left.100{ }^{\circ} \mathrm{C}\right),(\mathrm{V}) d=8.00 \mathrm{~nm}\left(s=0.125 \mathrm{~nm}^{-1}, 135^{\circ} \mathrm{C}\right)$. Two light vertical lines are drawn at $0{ }^{\circ} \mathrm{C}$ to illustrate the super cooling of water.

At ambient temperature, up to 4 equidistant reflections in the small angle scattering (SAXS) region but with only very weak intensities are present (see also SI, Figure S9 and Table S3). Thus, the lamellar structures formed are only poorly ordered in the $z$-direction. The determined repeat distance (membrane thickness plus inter-lamellar water layer thickness) is $d=6.66 \mathrm{~nm}$ 
and, hence, in the range of typical bolalipids ${ }^{17,24}$ forming monolayer lamellar structures. Compare to the molecule length of about $6.2 \mathrm{~nm}$, the observed repeat distance of $6.66 \mathrm{~nm}$ again indicates an monolayer lamellar phase with interdigitated chains as observed before for the bolalipid DMAPPC-C32-OH with unmodified chain but different headgroup ${ }^{24}$ and for an asymmetrical single-chain bolalipid with a shorter, unmodified alkyl chain (PC-C22-OH). ${ }^{46}$ However, for the water layer a thickness of only $0.46 \mathrm{~nm}$ would result. This is unlikely, as the PC headgroup is normally well hydrated. We therefore propose a tilting of the chains relative to the layer normal (Figure 6). This tilting of the chains would also lead to a better packing of the phenyl rings in the middle of the layer. Other asymmetrical bolalipids that derived from archaebacteria can also form interdigitated monolayer membranes. ${ }^{47}$

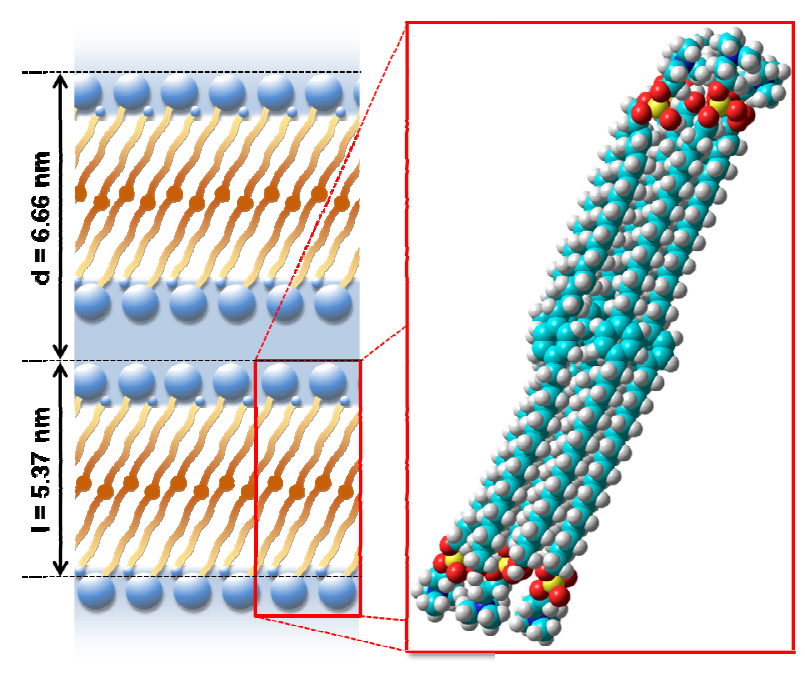

Figure 6. Left: Scheme of a lamellar phase consisting of interdigitated asymmetrical bolalipid molecules in a monolayer arrangement with a tilt of $30^{\circ}$ of the bolalipid molecules relative to the layer normal. This tilt is possibly caused by steric reasons to optimize packing for the phenyl rings. Right: CPK-model of seven $\mathrm{PC}-\mathrm{C} 17 \mathrm{pPhC} 17-\mathrm{OH}$ molecules in a monolayer arrangement.

In the wide angle X-ray scattering (WAXS) region, 6 sharp reflections appear which we could not index so far. The reflections indicate a well ordered almost crystalline-like packing of the alkyl chains. The WAXS-pattern could reflect a superposition of two individually scattering 
triclinic lattices (with three reflections for each lattice). More detailed studies on the indexing will be the focus of future work.

At $58{ }^{\circ} \mathrm{C}$ a change in scattering in the SAXS region occurs, only three reflections with much higher intensities are observable above the DSC transition. The position of the first order reflection is shifted to $s=0.158 \mathrm{~nm}^{-1}$ at $70{ }^{\circ} \mathrm{C}(d=6.32 \mathrm{~nm})$. A further increase in temperature leads to a further shift in the position of $s_{1}$ to $s=0.120 \mathrm{~nm}^{-1}(d=8.33 \mathrm{~nm})$ at $100{ }^{\circ} \mathrm{C}$ indicating an increase in lamellar repeat spacing probably due to an increase in water layer thickness.

In the WAXS region, heating to $58^{\circ} \mathrm{C}$, above the $T_{m}$ observed in the DSC, a drastic change of the scattering pattern occurs, as now, essentially only one reflection remains (see also Figure $\mathrm{S} 10)$. The maximum of the peak is located at $s_{1}=2.348 \mathrm{~nm}^{-1}$. Closer inspection shows that it might be superimposed on a broad reflection at $s_{2}=2.458 \mathrm{~nm}^{-1}$. This would indicate an $L_{\beta}$ chain packing mode. The FTIR spectra taken at temperatures above $58{ }^{\circ} \mathrm{C}$ showed that some disordering of the chains has already set in as the $\mathrm{CH}_{2}$-deformation band has shifted and the frequency of the $\mathrm{CH}_{2}$-stretching bands have also increased slightly (see above). It is therefore likely that the chains are tilted and the alkyl chain segments become rotationally disordered above $58{ }^{\circ} \mathrm{C}$.

In contrast to DSC or FT-IR measurements, the sample used for X-ray scattering experiments could be heated to high temperature as the capillary was sealed. Therefore, the "melting temperature" of the lamellar monolayer phase could be determined. At a temperature above $120^{\circ} \mathrm{C}$, the chains become finally disordered and a fluid lamellar phase is formed as indicated by the broad halo in the WAXS region caused by the scattering of disordered chains. The SAXS reflections at high temperature phase indicate the presence of a larger repeat distance $(\mathrm{s}=$ $0.125 \mathrm{~nm}^{-1}, d=8.00 \mathrm{~nm}$ ) though only two SAXS reflections are present and a lamellar lattice cannot be reliably indexed. This would indicate that the decrease in monolayer thickness due to 
the fluidization of the chains is overcompensated by an increase in water layer thickness.

For the interdigitated monolayer membrane of the previously investigated bolalipid DMAPPC-C32-OH with unmodified but effectively shorter chain we also found that the transition into the fluid lamellar phase occurred at very high temperature above $100{ }^{\circ} \mathrm{C} .{ }^{24}$ For a similar compound with only $22 \mathrm{CH}_{2}$-groups (PC-C22-OH) a transition into the fluid lamellar monolayer phase at a temperature of $80{ }^{\circ} \mathrm{C}$ was found. ${ }^{46}$ Therefore, it is clear that the transition into a fluid monolayer phase is dependent on the chain length. In the case of $\mathrm{PC}-\mathrm{C} 17 \mathrm{pPhC} 17-\mathrm{OH}$ the phenyl ring in the middle of the chain increases effectively the chain length, but on the other hand leads to a perturbation of the chain packing. Thus the observed "melting" temperature is somewhat lower than expected from the overall chain length but still higher than the other compounds with shorter chains.

The additional phenyl ring in the middle part of the alkyl chain of both bolalipids could be capable for pi stacking that possibly increases the interaction of different bola molecules. However, these interactions are short-ranged and further depend on the relative orientations of the benzene rings interacting with each other. This orientation is in turn basically determined by steric requirements of the $\mathrm{PC}$ headgroup area in comparison to the cross-sectional area of the single alkyl chain, which lead to a helical arrangement of symmetrical bola molecules within the nanofibers ${ }^{10}$ and, hence, the phenyl rings cannot come into a close contact for constructive pi stacking. For the asymmetrical bolalipids the pi stacking could become more likely since the alkyl chains can get into a closer contact due the smaller hydroxy headgroup and the resulting interdigitated arrangement of the bolalipid molecules within the monolayer (see CPK-model in Figure 6 right part). However, the contribution of pi stacking to the strength of intermolecular interaction is, in our view, small and the self-assembly of the bolalipids in lamellar aggregates 
and also nanofibers is mainly driven by hydrophobic (van-der-Waals) interactions of the long alkyl chain.

Packing parameter $P$ :

An explanation for the different aggregation behavior of both bolalipids, which is obviously related to the headgroups' asymmetry, could probably be found applying the packing parameter $(P)$. This parameter was introduced by Israelachvili ${ }^{48,49}$ to predict the aggregation behavior of classical monopolar amphiphiles in water. The adaptation of this geometrical approach from monopolar to bipolar lipids is problematic, since the single-chain bolalipids mostly have a stretched (all-trans) alkyl chain conformation and, hence, two "separated" headgroups rather than a $U$-shaped conformation bearing the two headgroups in a close contact. However, in a recent publication we applied this concept for bipolar lipids with a single unmodified C32-alkyl chain and symmetrical headgroups of different size. We could show that nanofibers are the stable aggregate form if the values of $P$ are in the range between 0.28 and $0.41 .^{13}$ If $P$ exceeds a value of 0.41 , the formation of lamellar or sheet-like aggregates is preferred. In the present case, the symmetrical PC-C17pPhC17-PC has a value of $P=0.40$ and the asymmetrical PC-C17pPhC17$\mathrm{OH}$ a value of $P=0.57$ (see Figure S11, Table S4 and SI for further details). These calculated $P$ values could hence be an explanation for the different aggregation behavior of both bolalipid in water although this value is only of minor significance for bipolar amphiphiles (see SI).

\section{Mixing behavior with classical phospholipids}

PC-C17pPhC17-PC with DPPC and DSPC:

For the mixing experiments of both bolalipids with bilayer forming phospholipids we chose the double-chain phospholipids DPPC and DSPC. Unmodified bolalipids, such as PC-C32-PC, did not incorporate into phospholipid bilayers of, e.g., DPPC, 1,2-dimyristoyl-sn-glycero-3- 
phosphocholine (DMPC), or 1-palmitoyl-2-oleoyl-sn-glycero-3-phosphocholine (POPC) due to packing frustration caused by the larger space requirement of the PC headgroup of PC-C32-PC in comparison to the small cross-sectional area of the alkyl chain. ${ }^{50}$ The additional phenylene ring in the middle part of the alkyl chain of both bolalipids investigated in this work increases the volume of the hydrocarbon chain, which could possibly improve the miscibility with phospholipids and, at best, result in a stabilized bilayer membrane.

We first investigated the thermotropic behavior of aqueous suspensions of equimolar mixtures of the symmetrical PC-C17pPhC17-PC with DPPC (Figure 7A) and DSPC (Figure 7B). In the case of pure DPPC and DSPC, the two well-known endothermic transitions are observed in the thermogram: the pre-transition from $L_{\beta^{\prime}}$ - to the $P_{\beta^{\prime}}$-phase (ripple phase) and the main transition at $41.9{ }^{\circ} \mathrm{C}(\mathrm{DPPC})$ and $54.9^{\circ} \mathrm{C}(\mathrm{DSPC})$ to the $L_{\alpha}$-phase. The pure PC-C17pPhC17-PC shows a fiber-micelle-transition at $22.8^{\circ} \mathrm{C}$. In an equimolar mixture of PC-C17pPhC17-PC with DPPC the DSC heating scan (Figure 7A) show two endothermic transitions between $2{ }^{\circ} \mathrm{C}$ and $80{ }^{\circ} \mathrm{C}$ : a broad transition at $T=29-30{ }^{\circ} \mathrm{C}$ and a second one slightly below the DPPC main transition with a splitting $\left(40.4{ }^{\circ} \mathrm{C}, 41.3{ }^{\circ} \mathrm{C}\right)$. The corresponding cooling curve shows three peaks at $39.9^{\circ} \mathrm{C}$, $34.6{ }^{\circ} \mathrm{C}$, and $30.0{ }^{\circ} \mathrm{C}$, whereas the cooling scan of the pure bolalipid shows no transition due to the slow kinetics for the reformation of the nanofibers as already described above.

Similar results were found for the equimolar mixture of $\mathrm{PC}-\mathrm{C} 17 \mathrm{pPhC} 17-\mathrm{PC}$ with the longer chain DSPC. In the DSC heating scan (Figure 7B) we observe two large endothermic transitions at $53.5^{\circ} \mathrm{C}$ and $54.9^{\circ} \mathrm{C}$ together with two small peaks at $24.2^{\circ} \mathrm{C}$ and $41.0^{\circ} \mathrm{C}$. The corresponding cooling scan reveals several transition peaks, the most distinctive ones at $53.6{ }^{\circ} \mathrm{C}, 51.8^{\circ} \mathrm{C}$, and $48.3{ }^{\circ} \mathrm{C}$.

In addition, the DSC heating scan of the DPPC-mixture shows a reproducible, exothermic 
transition peak at about $60{ }^{\circ} \mathrm{C}$. For the corresponding DSPC-mixture only a decrease in the heat capacity beginning at $70{ }^{\circ} \mathrm{C}$ is seen. The origin of this effect remains unclear at this time, but it is conceivable that a metastable state is reached after the main transition, which further transforms within the timescale of the DSC experiment (heating rate $=60 \mathrm{~K} \mathrm{~h}^{-1}$ ) into a thermodynamically stable state indicated by this exothermic transition. The same effect was found earlier for a mixture of DPPC with PC-C32-10,10'Me-PC - a bolalipid with two methyl groups in the chain. ${ }^{13}$
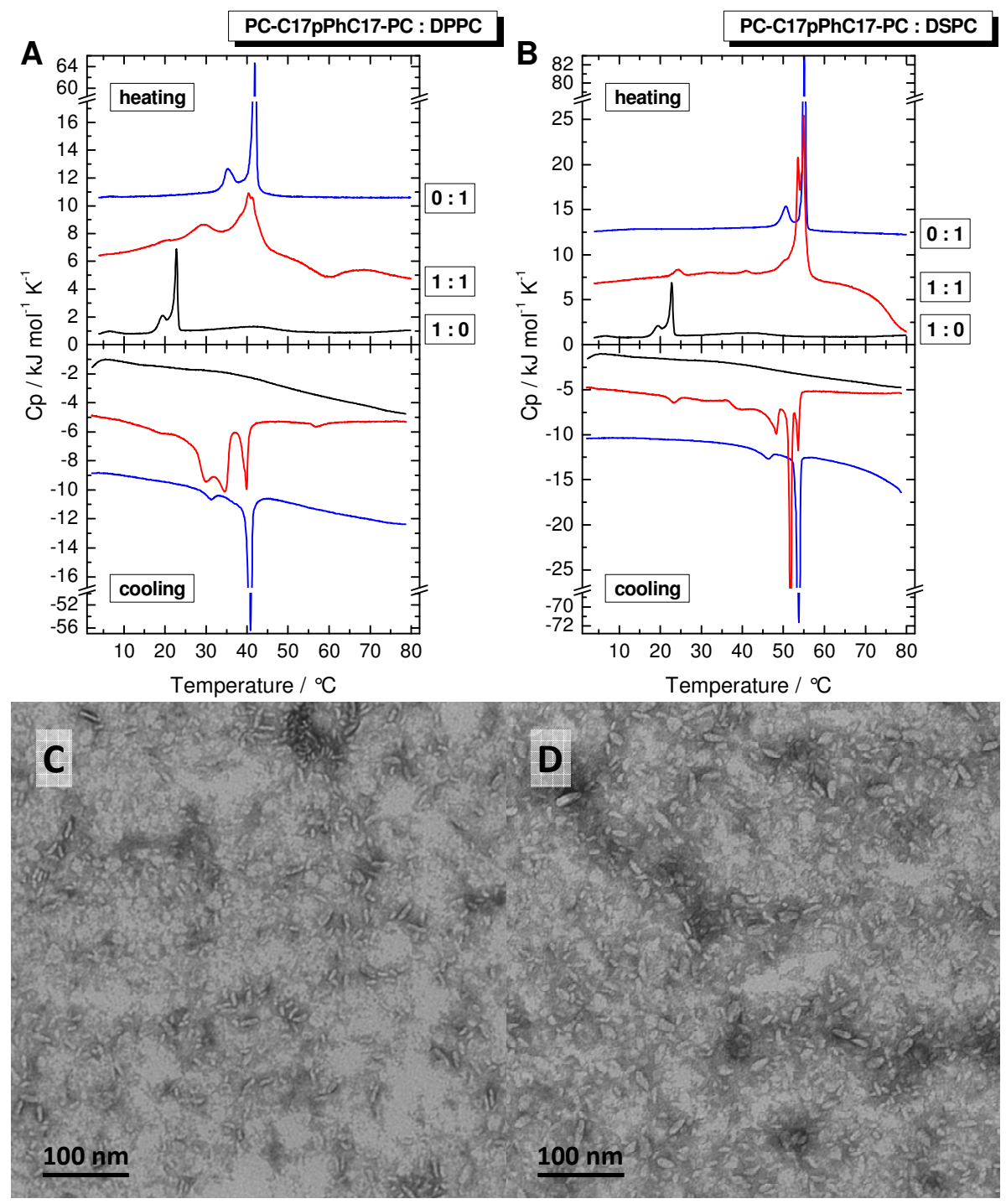

Figure 7. A,B: DSC heating and cooling curves of equimolar mixtures $(c=3 \mathrm{mM})$ of $\mathrm{PC}$ C17pPhC17-PC with DPPC (A) and DSPC (B) in phosphate buffer solution (10 mM, pH = 7.4). DSC curves of pure compounds are presented for comparison. The curves are shifted vertically 
for clarity. C,D: TEM images of equimolar mixtures $(c=60 \mu \mathrm{M})$ of PC-C17pPhC17-PC with (C) DPPC and (D) DSPC in phosphate buffer $(10 \mathrm{mM}, \mathrm{pH}=7.4)$. The samples were prepared at room temperature and negatively stained with uranyl acetate.

To get an idea about the shape of the aggregates formed in aqueous suspension TEM images were recorded from negatively stained samples. All samples were prepared at room temperature, i.e., below the transitions observed in DSC. The images are shown in Figure 7C,D. In both cases, no closed lipid vesicles (liposomes) can be observed as found for pure DPPC and DSPC in aqueous suspension. Instead, small elongated micelles and small bilayer fragments are present. These aggregates have a size of about $20-30 \mathrm{~nm}$ in length and in the case of bilayer fragments they are either oriented parallel or perpendicular to the surface of the grid. We can only speculate about the composition of these mixed lipid aggregates, but it seems clear from the images and also from the DSC curves that a partial demixing occurs. The elongated micelles contain probably mostly bolalipids with some phospholipids and the bilayer disks contain some bolalipids probably enriched at the rim of the phospholipid bilayer fragments.

Based on the DSC data, the TEM images, and the fact that both bolalipid: phospholipid mixtures are clear and transparent solutions (without the use of sonication) we can conclude that we indeed have a partial miscibility of both components, which is in contrast to mixtures of the unmodified PC-C32-PC with DPPC. In the latter case, EM images showed a coexistence of long PC-C32-PC nanofibers and DPPC vesicles. ${ }^{50}$

\section{PC-C17pPhC17-OH with DPPC:}

In the second series of mixing experiments we used the asymmetrical $\mathrm{PC}-\mathrm{C} 17 \mathrm{pPhC} 17-\mathrm{OH}$ together with DPPC. Since PC-C17pPhC17-OH forms lamellar aggregates by itself, the possibility exists that this asymmetrical bolalipid can be incorporated into DPPC bilayers leading to a stabilization of the DPPC lipid membranes. The temperature dependent aggregation behavior of 
PC-C17pPhC17-OH : DPPC mixtures at different molar ratios was again investigated by calorimetric measurements (see Figure 8).

The DSC heating scans in Figure $8 \mathrm{~A}$ show that $T_{m}$ of DPPC at $41.9^{\circ} \mathrm{C}$ is not affected by an increasing amount of the asymmetrical bolalipid. The transition peak of $\mathrm{PC}-\mathrm{C} 17 \mathrm{pPhC} 17-\mathrm{OH}$ is slightly shifted to higher temperatures (by $1.2 \mathrm{~K}$ to $54.6{ }^{\circ} \mathrm{C}$ ) in mixtures with DPPC. In addition, a second endothermic transition occurs at $57.8^{\circ} \mathrm{C}$. Both transition peaks stay at the same temperature independent of the mixing ratio. In the cooling scans only two peaks are observed (Figure 8B): $T_{m}$ of DPPC is again not influenced by the bolalipid and the PC-C17pPhC17-OH transition is shifted to slightly higher temperatures by $1.3 \mathrm{~K}$ compared to the pure bolalipid but in this case no additional transition is observed. Again, both transitions stay at the same temperature for each molar ratio investigated, only that the relative peak areas change according to the mixing ratio.

The DSC curves clearly indicate that the asymmetrical bolalipid PC-C17pPhC17-OH cannot be incorporated into DPPC bilayer membranes. These two lipids seem to be almost completely immiscible despite the fact that both form lamellar phases, DPPC a bilayer phase and PC$\mathrm{C} 17 \mathrm{pPhC17-OH}$ a monolayer lamellar phase. The small increase in the main transition temperature of $\mathrm{PC}-\mathrm{C} 17 \mathrm{pPhC} 17-\mathrm{OH}$ in its mixtures with $\mathrm{DPPC}$ indicates that possibly a small amount of DPPC can be inserted in the lamellar monolayer structures of the bolalipid. Likewise the slight broadening of the main transition of DPPC indicates some perturbation of the packing due to incorporation of the bolalipid into the bilayer membranes. 

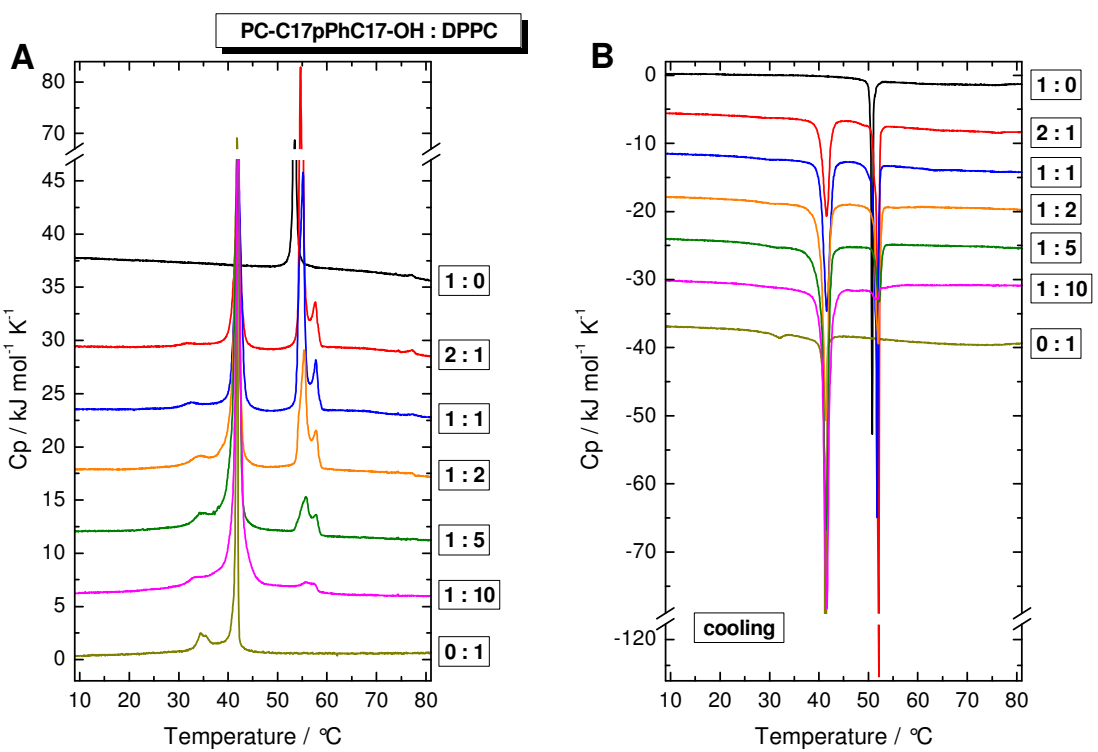

Figure 8. DSC curves of $3 \mathrm{mM}$ suspensions of $\mathrm{PC}-\mathrm{C} 17 \mathrm{pPhC} 17-\mathrm{OH}: \mathrm{DPPC}$ mixtures in phosphate buffer solution $(10 \mathrm{mM}, \mathrm{pH}=7.4)$ with different molar rations: (A) heating, (B) cooling. The heating/cooling rate was $20 \mathrm{~K} \mathrm{~h}^{-1}$. The curves are shifted vertically for clarity.

These findings are supported by TEM images (Figure 9) of uranyl acetate stained suspensions of PC-C17pPhC17-OH : DPPC mixtures that show the presence of two different aggregate types: on the one hand closed bilayer vesicles formed of DPPC and on the other hand large lamellar aggregates composed of the asymmetrical bolalipid. The immiscibility is probably due to the fact that $\mathrm{PC}-\mathrm{C} 17 \mathrm{pPhC} 17-\mathrm{OH}$ forms a very stable monolayer lamellar phase, which becomes fluid only at very high temperature above $120{ }^{\circ} \mathrm{C}$ (see above). This is energetically favored over the incorporation into DPPC bilayer membranes as in this case the formation of void volume cannot be avoided when $\mathrm{PC}-\mathrm{C} 17 \mathrm{pPhC} 17-\mathrm{OH}$ is in a stretched conformation. This is due to packing problems caused by the large space requirement of the $\mathrm{PC}$ headgroup of $\mathrm{PC}-\mathrm{C} 17 \mathrm{pPhC} 17-\mathrm{OH}$ compared to the small cross-sectional area of the alkyl chain. Thus, the $\mathrm{PC}-\mathrm{C} 17 \mathrm{pPhC} 17-\mathrm{OH}$ prefers to self-assemble into separate lamellar aggregates, where the alkyl chain of the bolalipid can get in very close contact. 


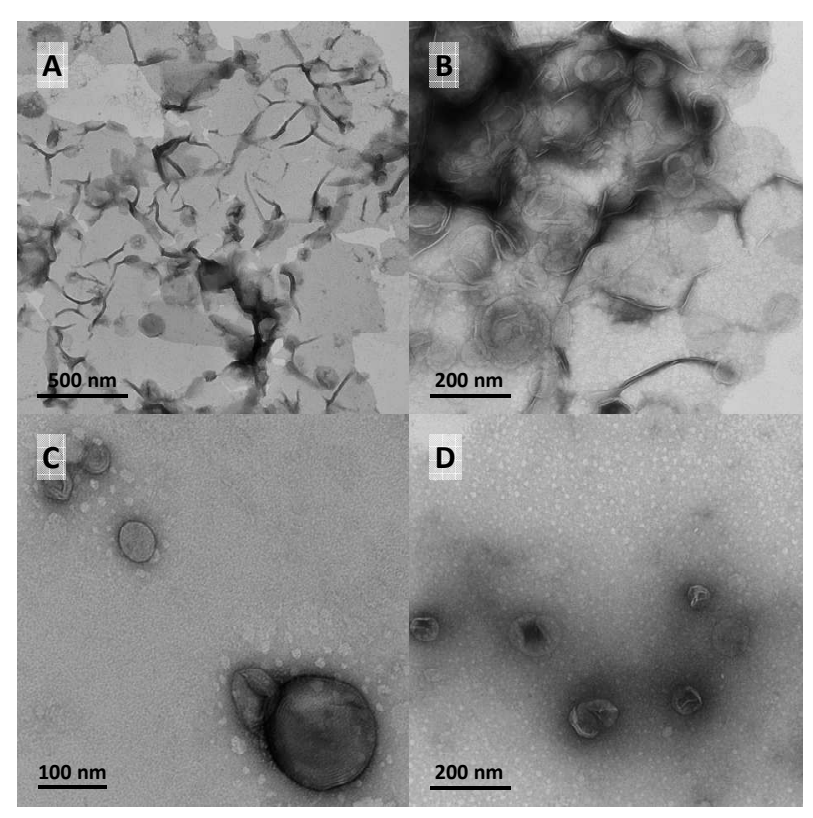

Figure 9. TEM images of PC-C17pPhC17-OH:DPPC mixtures ( $c=60 \mu \mathrm{M}$ in phosphate buffer solution $10 \mathrm{mM}, \mathrm{pH}=7.4$ ) with different molar ratios: A) $2: 1$, B) $1: 1$, C) $1: 5$ and D) $1: 10$. The samples were prepared at room temperature and stained with uranyl acetate solution.

\section{CONCLUSIONS}

Two single-chain phenylene-modified bolalipids with either two phosphocholine headgroups resulting in a symmetrical example (PC-C17pPhC17-PC) or a phosphocholine and a hydroxy headgroup leading to the asymmetrical counterpart (PC-C17pPhC17-OH) were synthesized using a stepwise Sonogashira cross-coupling reaction of dibromo benzene derivatives with various, orthogonally protected alkynols as key step.

The aggregation behavior of both bolalipids in aqueous suspension as a function of temperature showed that depending on the headgroup asymmetry, nanofibers or lamellar aggregates are formed: The symmetrical PC-C17pPhC17-PC self-assembles into nanofibers with a diameter of $5.7 \mathrm{~nm}$ corresponding to the length of bolalipid molecule. Physical cross-linking and entanglements of the fibers lead to a gelation of water. However, this hydrogel is less stable compared to a hydrogel composed of unmodified bolalipids, e.g., PC-C32-PC, due to the 
additional phenyl ring within the alkyl chain. An increase in temperature leads to the reversible transformation of the nanofibers into small elongated micelles.

The asymmetrical $\mathrm{PC}-\mathrm{C} 17 \mathrm{pPhC} 17-\mathrm{OH}$ self-assembles in aqueous suspension into large lamellar structures. The thickness of the lamellae is $6.66 \mathrm{~nm}$ indicating a monolayer of molecules with interdigitated alkyl chains. The alkyl chains are in an all-trans conformation, closely packed and possibly tilted relative to the layer normal. An increase in temperature above $58{ }^{\circ} \mathrm{C}$ causes a change in the packing mode and the chains become probably rotationally disordered however retaining their all-trans conformation up to very high temperatures of about $120^{\circ} \mathrm{C}$. Above this temperature a melting of the alkyl chains into liquid-crystalline phases occurs.

The mixing behavior of these novel phenylene-modified bolalipids with bilayer forming phospholipids, e.g., DPPC and DSPC, was studied by DSC and by TEM. We observed a completely different mixing behavior of both bolalipids depending on the headgroup asymmetry: The symmetrical PC-C17pPhC17-PC is partially miscible with DPPC and DSPC, respectively, however, closed lipid vesicles are not observed and bilayer fragments as well as elongated micelles are formed instead. The main phase transitions of DPPC or DSPC are slightly broadened but not significantly shifted indicating that no stabilization effect of the bilayers by the bolalipid occurs. The asymmetrical PC-C17pPhC17-OH shows almost complete immiscibility with DPPC at all DPPC : PC-C17pPhC17-OH ratios despite the fact that both components self-assemble in aqueous suspensions in lamellar structures. This is indicated by the DSC scans showing two independent endothermic transitions that belong to the pure compounds and by the TEM images, where two types of aggregates, closed vesicles of DPPC and large lamellae of the bolalipid are seen. The results show that a delicate balance between steric requirements of the headgroups and alkyl chains and intermolecular interactions in these long-chain compounds 
leads to quite unexpected mixing behavior with classical phospholipids forming lipid bilayer phases.

\section{ASSOCIATED CONTENT}

Supporting Information. Synthetic procedures, analytical data, ESI-MS, ${ }^{1} \mathrm{H}-,{ }^{13} \mathrm{C}-\mathrm{NMR}$, and HRMS spectra of prepared compounds, additional DSC, FTIR, and TEM measurements, further SANS and X-ray scattering data. This material is available free of charge via the Internet at http://pubs.acs.org.

\section{AUTHOR INFORMATION}

\section{Corresponding Author}

*simon.drescher@pharmazie.uni-halle.de, phone: +49-345-5525196, fax: +49-345-5527026.

\section{Author Contributions}

The manuscript was written by contributions of all authors. All authors have given approval to the final version of the manuscript.

\section{ACKNOWLEDGMENT}

This work was financially supported by grants (project B1 182/19-3 to S.D., A.M., and A.B.) and by grants within to Forschergruppe FOR 1145 (to B.-D.L. and A.B.) from the Deutsche Forschungsgemeinschaft (DFG). This research project has also been supported by the European Commission under the 7th Framework Programmes through the 'Research Infrastructures' action of the 'Capacities Programme' Contact No: CP-CSA_INFRA-2008-1.1.1 Number 226507NMI3. The support of Dr Gerd Hause (Biocenter, MLU Halle-Wittenberg) by providing us access to the electron microscope facility is greatly appreciated. Finally, S.D. thanks Prof Andrea 
Sinz and Dr Christian Ihling (Department of Pharmaceutical Chemistry and Bioanalytics, MLU Halle-Wittenberg) for the high resolution mass spectrometry analysis.

\section{REFERENCES}

(1) Fuhrhop, J.-H.; Wang, T. Bolaamphiphiles. Chem. Rev. 2004, 104, 2901-2937.

(2) Langworthy, T. A. Long-chain diglycerol tetraethers from Thermoplasma acidophilum. Biochim. Biophys. Acta, Lipids Lipid Metab. 1977, 487, 37-50.

(3) De Rosa, M.; Esposito, E.; Gambacorta, A.; Nicolaus, B.; Bu'Lock, J. D. Effects of temperature on ether lipid composition of Caldariella acidophila. Phytochemistry 1980, 19, 827831.

(4) Gambacorta, A.; Gliozzi, A.; Rosa, M. Archaeal lipids and their biotechnological applications. World J. Microb. Biot. 1995, 11, 115-131.

(5) Estroff, L. a.; Hamilton, A. D. Water gelation by small organic molecules. Chem. Rev. 2004, $104,1201-1218$.

(6) Benvegnu, T.; Brard, M.; Plusquellec, D. Archaeabacteria bipolar lipid analogues: structure, synthesis and lyotropic properties. Curr. Opin. Colloid Interface Sci. 2004, 8, 469-479.

(7) Benvegnu, T.; Lemiègre, L.; Cammas-Marion, S. Archaeal Lipids: Innovative Materials for Biotechnological Applications. Eur. J. Org. Chem. 2008, 2008, 4725-4744.

(8) Yan, Y.; Lu, T.; Huang, J. Recent advances in the mixed systems of bolaamphiphiles and oppositely charged conventional surfactants. J. Colloid Interf. Sci. 2009, 337, 1-10.

(9) Chong, P. L.-G. Archaebacterial bipolar tetraether lipids: Physico-chemical and membrane properties. Chem. Phys. Lipids 2010, 163, 253-265.

(10) Köhler, K.; Förster, G.; Hauser, A.; Dobner, B.; Heiser, U. F.; Ziethe, F.; Richter, W.; Steiniger, F.; Drechsler, M.; Stettin, H.; Blume, A. Temperature-dependent behavior of a 
symmetric long-chain bolaamphiphile with phosphocholine headgroups in water: from hydrogel to nanoparticles. J. Am. Chem. Soc. 2004, 126, 16804-16813.

(11) Meister, A.; Drescher, S.; Mey, I.; Wahab, M.; Graf, G.; Garamus, V. M.; Hause, G.; Mögel, H.-J.; Janshoff, A.; Dobner, B.; Blume, A. Helical nanofibers of self-assembled bipolar phospholipids as template for gold nanoparticles. J. Phys. Chem. B 2008, 112, 4506-4511.

(12) Wahab, M.; Schiller, P.; Schmidt, R.; Moegel, H. J. Monte Carlo Study of the Self-Assembly of Achiral Bolaform Amphiphiles into Helical Nanofibers. Langmuir 2010, 26, 2979-2982.

(13) Blume, A.; Drescher, S.; Meister, A.; Graf, G.; Dobner, B. Tuning the Aggregation Behaviour of Single-Chain Bolaphospholipids in Aqueous Suspension: From Nanoparticles to Nanofibres to Lamellar Phases. Faraday Disc. 2013, 161, 193-213.

(14) Drescher, S.; Meister, A.; Blume, A.; Karlsson, G.; Almgren, M.; Dobner, B. General synthesis and aggregation behaviour of a series of single-chain 1, $\omega$-bis(phosphocholines). Chem. Eur. J. 2007, 13, 5300-5307.

(15) Graf, G.; Drescher, S.; Meister, A.; Garamus, V. M.; Dobner, B.; Blume, A. Bolalipid fiber aggregation can be modulated by the introduction of sulfur atoms into the spacer chains. J. Coll. Interf. Sci. 2013, 393, 143-150.

(16) Meister, A.; Bastrop, M.; Koschoreck, S.; Garamus, V. M.; Sinemus, T.; Hempel, G.; Drescher, S.; Dobner, B.; Richtering, W.; Huber, K.; Blume, A. Structure-property relationship in stimulus-responsive bolaamphiphile hydrogels. Langmuir 2007, 23, 7715-7723.

(17) Meister, A.; Drescher, S.; Karlsson, G.; Hause, G.; Baumeister, U.; Hempel, G.; Garamus, V. M.; Dobner, B.; Blume, A. Formation of square lamellae by self-assembly of long-chain bolaphospholipids in water. Soft Matter 2010, 6, 1317-1324. 
(18) Meister, A.; Drescher, S.; Garamus, V. M.; Karlsson, G.; Graf, G.; Dobner, B.; Blume, A. Temperature-dependent self-assembly and mixing behavior of symmetrical single-chain bolaamphiphiles. Langmuir 2008, 24, 6238-6246.

(19) Fuhrhop, J.-H.; Spiroski, D.; Boettcher, C. Molecular monolayer rods and tubules made of $\alpha$-(L-lysine), $\omega$-(amino) bolaamphiphiles. J. Am. Chem. Soc. 1993, 115, 1600-1601.

(20) Masuda, M.; Shimizu, T. Lipid nanotubes and microtubes: experimental evidence for unsymmetrical monolayer membrane formation from unsymmetrical bolaamphiphiles. Langmuir 2004, 20, 5969-5977.

(21) Kameta, N.; Masuda, M.; Minamikawa, H.; Mishima, Y.; Yamashita, I.; Shimizu, T. Functionalizable Organic Nanochannels Based on Lipid Nanotubes: Encapsulation and nanofluidic Behavior of Biomacromolecules. Chem. Mater. 2007, 19, 3553-3560.

(22) Kameta, N.; Masuda, M.; Minamikawa, H.; Shimizu, T. Self-Assembly and Thermal Phase Transition Behavior of Unsymmetrical Bolaamphiphiles Having Glucose- and AminoHydrophilic Headgroups. Langmuir 2007, 23, 4634-4641.

(23) Masuda, M.; Shimizu, T. Multilayer structure of an unsymmetrical monolayer lipid membrane with a 'head-to-tail' interface. Chem. Commun. 2001, 2442-2443.

(24) Graf, G.; Drescher, S.; Meister, A.; Garamus, V. M.; Dobner, B.; Blume, A. Tuning the Aggregation Behaviour of Single-Chain Bolaamphiphiles in Aqueous Suspension by Changes in Headgroup Asymmetry. Soft Matter 2013, 9, 9562-9571.

(25) Gabriel, J. L.; Chong, P. L. Molecular modeling of archaebacterial bipolar tetraether lipid membranes. Chem. Phys. Lipids 2000, 105, 193-200.

(26) Gu, Q.; Zou, A.; Yuan, C.; Guo, R. Effects of a bolaamphiphile on the structure of phosphatidylcholine liposomes. J. Colloid Interf. Sci. 2003, 266, 442-447. 
(27) Fuhrhop, J. H.; Liman, U.; Koesling, V. A Macrocyclic Tetraether Bolaamphiphile and an Oligoamino $\alpha, \omega$-Dicarboxylate Combine To Form Monolayered, Porous Vesicle Membranes, Which Are Reversibly Sealed by EDTA and Other Bulky Anions. J. Am. Chem. Soc. 1988, 110, 6840-6845.

(28) Moss, R. A.; Li, G.; Li, J.-M. Enhanced Dynamic Stability of Macrocyclic and Bolaamphiphilic Macrocyclic Lipids in Liposomes. J. Am. Chem. Soc. 1994, 116, 805-806.

(29) Sprott, G. D.; Tolson, D. L.; Patel, G. B. Archaeosomes as novel antigen delivery systems. FEMS Microbiol. Lett. 1997, 154, 17-22.

(30) Benvegnu, T.; Réthoré, G.; Brard, M.; Richter, W.; Plusquellec, D. Archaeosomes based on novel synthetic tetraether-type lipids for the development of oral delivery systems. Chem. Commun. 2005, 5536-5538.

(31) Jacquemet, A.; Barbeau, J.; Lemiègre, L.; Benvegnu, T. Archaeal tetraether bipolar lipids: Structures, functions and applications. Biochimie 2009, 91, 711-717.

(32) Grinberg, S.; Kipnis, N.; Linder, C.; Kolot, V.; Heldman, E. Asymmetric bolaamphiphiles from vernonia oil designed for drug delivery. Eur. J. Lipid Sci. Technol. 2010, 112, 137-151.

(33) Nuraje, N.; Bai, H.; Su, K. Bolaamphiphilic molecules: Assembly and applications. Progress in Polymer Science 2013, 38, 302-343.

(34) Philosof-Mazor, L.; Dakwar, G. R.; Popov, M.; Kolusheva, S.; Shames, A.; Linder, C.; Greenberg, S.; Heldman, E.; Stepensky, D.; Jelinek, R. Bolaamphiphilic vesicles encapsulating iron oxide nanoparticles: New vehicles for magnetically targeted drug delivery. Int. J. Pharm. 2013, 450, 241-249.

(35) Rosta, L. Cold neutron research facility at the Budapest Neutron Centre. Appl. Phys. A 2002, 74, 292-294. 
(36) Drescher, S.; Becker, S.; Dobner, B.; Blume, A. Bis-Sonogashira Cross-Coupling: An Expeditious Approach towards Long-Chain, Phenylene-Modified 1, $\omega$-Diols. RSC Adv. 2012, 2, $4052-4054$.

(37) Drescher, S.; Sonnenberger, S.; Meister, A.; Blume, A.; Dobner, B. Synthesis and Aggregation Behaviour of Symmetrical Phenylene/Biphenylene-modified Bolaamphiphiles. Monatsh. Chem. 2012, 143, 1533-1543.

(38) Mantsch, H. H.; McElhaney, R. N. Phospholipid phase transitions in model and biological membranes as studied by infrared spectroscopy. Chem. Phys. Lipids 1991, 57, 213-226.

(39) Mendelsohn, R.; Moore, D. J. Vibrational spectroscopic studies of lipid domains in biomembranes and model systems. Chem. Phys. Lipids 1998, 96, 141-157.

(40) Snyder, R. G. Vibrational spectra of crystalline n-paraffins II. Intermolecular effects. J. Mol. Spectrosc. 1961, 7, 116-144.

(41) Snyder, R. G. Vibrational correlation splitting and chain packing for the crystalline alkanes. J. Chem. Phys. 1979, 71, 3229-3235.

(42) Lewis, R. N. A. H.; McElhaney, R. N. Studies of Mixed-Chain Diacyl Phosphatidylcholines with Highly Asymmetric Acyl Chains: A Fourier Transform Infrared Spectroscopic Study of Interfacial Hydration and Hydrocarbon Chain Packing in the Mixed lnterdigitated Gel Phase. Biophys. J. 1993, 65, 1866-1877.

(43) Wong, P. T. T. Pressure-induced Correlation Field Splitting of Vibrational Modes: Structural and Dynamic Properties in Lipid Bilayers and Biomembranes. Biophys. J. 1994, 66, 1505-1514. (44) Glatter, O. A New Method for the Evaluation of Small-Angle Scattering Data. J. Appl. Cryst. 1977, 10, 415-421. 
(45) Pedersen, J. S. Analysis of small-angle scattering data from colloids and polymer solutions: modeling and least-squares fitting. Adv. Colloid Interf. Sci. 1997, 70, 171-210.

(46) Pohle, W.; Selle, C.; Rettig, W.; Heiser, U.; Dobner, B.; Wartewig, S. Phase transitions and hydrogen bonding in a bipolar phosphocholine evidenced by calorimetry and vibrational spectroscopy. Arch. Biochem. Biophys. 2001, 396, 151-161.

(47) Gliozzi, A., In Handbook of Nonmedical Applications of Liposomes, Barenholz, Y.; Lasic, D. D., Eds. CRC Press: Boca Raton, New York/London/Tokyo, 1996; Vol. II, p 329.

(48) Israelachvili, J. N.; Marcelja, S.; Horn, R. G. Physical principles of membrane organisation. Quart. Rev. Biophys. 1980, 13, 121-200.

(49) Israelachvili, J. N., Intermolecular and Surface Forces. 3rd ed.; Academic Press: Burlington, 2011.

(50) Meister, A.; Köhler, K.; Drescher, S.; Dobner, B.; Karlsson, G.; Edwards, K.; Hause, G.; Blume, A. Mixing behaviour of a symmetrical single-chain bolaamphiphile with phospholipids. Soft Matter 2007, 3, 1025-1031. 


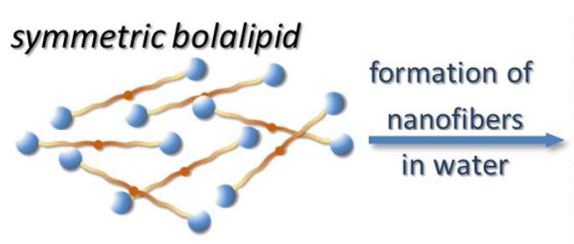

\section{asymmetric bolalipid}

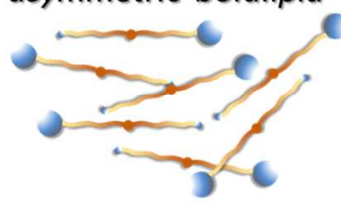

formation of monolayer membranes in water

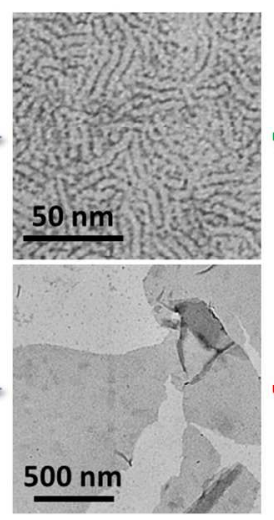

1:1 mixture with DPPC:

miscible
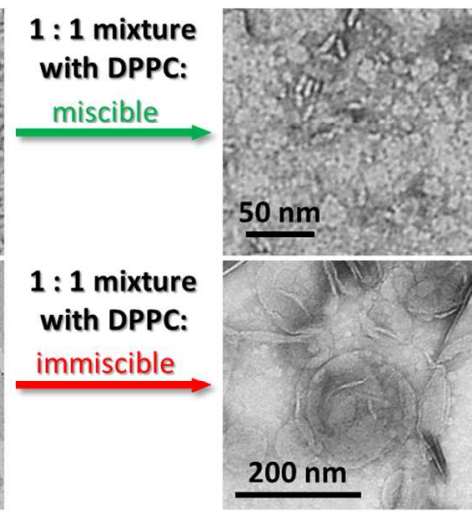